\title{
Operationalizing the emergent management of psychiatric patients during the COVID-19 pandemic at an academic public hospital
}

\author{
Ynhi Thomas*1, Sara Andrabi ${ }^{1}$, Nidal Moukaddam ${ }^{2}$, Asim Shah $^{2}$, Greg Buehler ${ }^{1}$, Jennifer Carnell ${ }^{1}$, Richina Bicette ${ }^{1}$, \\ Cedric Dark ${ }^{1}$, Sarah Bezek ${ }^{1}$ \\ ${ }^{1}$ Henry J.N. Taub Department of Emergency Medicine, Baylor College of Medicine, Houston, United States \\ ${ }^{2}$ Menninger Department of Psychiatry and Behavioral Sciences, Baylor College of Medicine, Houston, United States
}

Received: November 2, 2021

DOI: $10.5430 /$ jha.v10n5p24
Accepted: December 6, 2021

Online Published: December 13, 2021

\begin{abstract}
Background: While the volume of Emergency Department (ED) visits has declined during the 2019 novel coronavirus disease or COVID-19, the opposite has been observed with mental-health related visits. The need to screen and manage potential COVID-19 symptoms in parallel with psychiatric complaints have imposed new challenges in the ED at an academic public hospital.

Objective: The objective is to share operational modifications in addressing the challenges related to the influx of ED mentalhealth related complaints at the departmental, hospital-wide, and city-wide level within an academic, public hospital.

Methods: At the departmental level, a triage algorithm for screening patients with concerning symptoms was developed. A dedicated Respiratory Decision Unit with psychiatrically safe rooms that adhered to infection prevention protocol was also created. All staff were trained to utilize personal protective equipment through lectures, asynchronous learning, and multidisciplinary simulations. The ED team worked with hospital leadership to increase inpatient medical-psychiatric bed capacity and to develop testing protocols for patients being admitted to allow for cohorting of symptomatic patients. At the city level, leadership within the three main organizations that provide mental health services to the city met regularly to address operational issues.

Conclusions: The COVID-19 pandemic has imposed new challenges in terms of increased psychosocial needs while limiting transmission risks. Based on the experiences shared, a multitier approach is necessary. At all levels, the goals were to screen appropriately, decrease transmission risk, and maintain throughput. The purpose of this descriptive manuscript is to encourage dialogue and to raise awareness about the unique needs of the mental health system.
\end{abstract}

Key Words: Psychiatric emergencies, COVID-19, Mental health, ED operations, ED throughput

\section{INTRODUCTION}

While the COVID-19 pandemic has led to a decrease in the volume of Emergency Department (ED) visits by approximately $40 \%$ nationally, ${ }^{[1]}$ the opposite has been observed in terms of mental-health related visits. ${ }^{[2-4]}$ Reasons for the increase in utilization of the ED for mental-health complaints may be multifactorial. During the pandemic, there has been less use of non-acute mental health services, and the number of elective psychiatric admissions have also declined. ${ }^{[5]}$ Patients have noted difficulty in contacting their outpatient therapists or psychiatrists. More than one in three patients

*Correspondence: Ynhi Thomas; Email: ythomas@bcm.edu; Address: Henry J.N. Taub Department of Emergency Medicine, Baylor College of Medicine, Houston, United States. 
have also reported disruptions in accessing treatment or recovery support services for addiction. ${ }^{[6]}$ The pandemic has additionally created stressors including the general fear of getting sick, uncertainties with employment, financial strains, and social isolation that have acted as precipitating factors, causing an increased number of mental-health related emergencies. ${ }^{[4,7-10]}$

Operational changes to a public academic hospital ED were made due to the influx of ED psychiatric visits. The need to screen and manage potential COVID-19 infections in parallel with psychiatric symptoms has imposed new challenges to the care of these patients within the ED.

Even before the pandemic, the lack of mental health resources has been a strain on the United States medical system for years, especially for vulnerable patient populations such as the under or uninsured. ${ }^{[1-14]}$ The pandemic has only potentiated this problem.

Descriptive trends in ED psychiatric visits at a public academic hospital during the COVID-19 pandemic will first be provided. Next, there will be a discussion on the inter- and intra-hospital system modifications that were implemented in addressing the increased number of mental-health related emergency visits, highlighting how the interventions have improved patient care and operational efficiency. In addition, changes made to ensure the safety of both patients and staff during the pandemic will be highlighted.

Before discussing the general trends and the operational changes that were made, background information on the unique aspects of the ED being discussed will be provided. There is a dedicated psychiatry team within the ED, allowing for close collaboration and co-management of ED psychiatric patients. The emergency psychiatry team also oversees a locked unit within the ED, which is the only dedicated psychiatric ED within the surrounding metropolitan area. For patients with mental health complaints awaiting medical clearance, there is a close observation pod with dedicated patient care assistants and security staff for safety. All beds in the close observation pod are grouped in a single, large room and are partitioned by curtains. There is also a dedicated pod called the Respiratory Decision Unit (RDU) for patients under investigation (PUIs) or patients with possible or confirmed COVID-19 infection. The RDU has private rooms and is in a section of the ED physically isolated from non-PUI patients and the psychiatric close observation pod.

\section{Discussion}

\subsection{Trends in emergency psychiatric patient visits dur- ing the COVID-19 pandemic}

Data obtained for the following descriptive analyses were from existing monthly operational metrics reported for standard of care. The data was provided de-identified by the Psychiatry and Emergency Medicine Administrative Teams who received the information for quality tracking. No individual charts or personal health information were reviewed. As a result of these conditions, the Institutional Review Board (IRB) deemed that this project did not constitute human subjects research, and it did not fall under the regulations for IRB review.
A

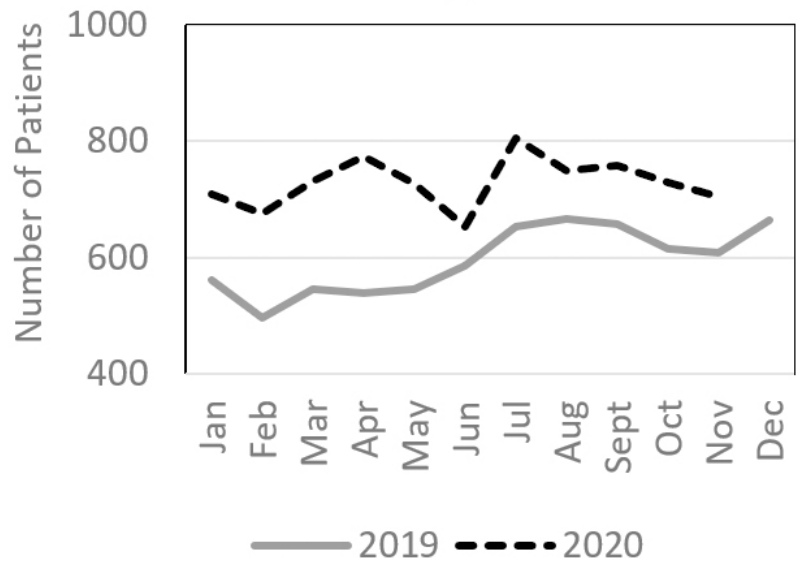

B

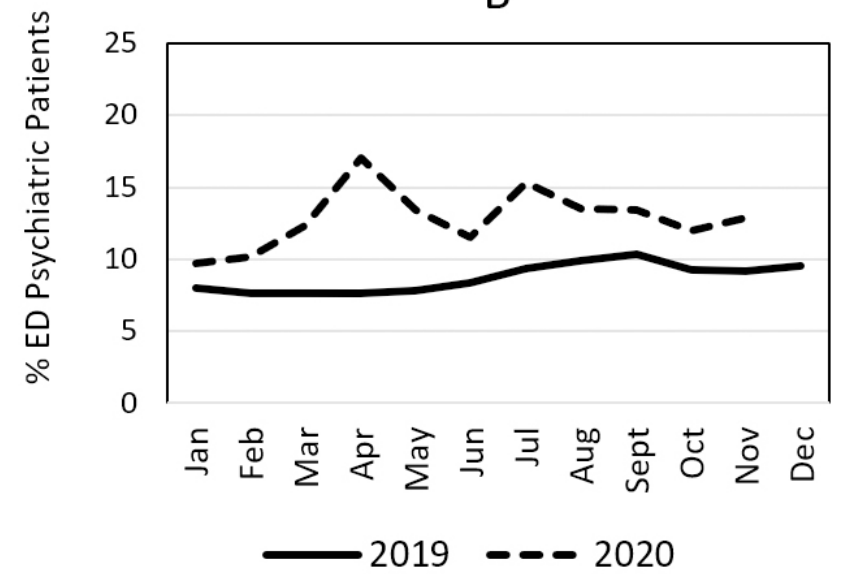

Figure 1. Figure 1A shows the total number of patients who presented to an academic public hospital ED within a major U.S. city in 2019 pre-pandemic and 2020 during the pandemic with mental-health related complaints. Figure 1B shows the percentage of these patients in relations to the total patients triaged into the ED. 
A rise in the number of visits for mental-health related complaints during the pandemic was noted, even though overall ED volume decreased. Figures $1 \mathrm{~A}$ and $1 \mathrm{~B}$ show the total volume and percentage of patients with mental-health related complaints were higher in 2020, including during the pandemic, compared to 2019. Patients also presented in extremis and were more likely to be brought in by police. This illustrates how patients likely delayed treatment for certain medical conditions, while presenting more emergently for acute mental health reasons.

The impact on ED operations was not significant during the early days of the pandemic within the country due to shutdown of non-essential businesses and a national stay-home order in March 2020. ${ }^{[15]}$ The stay-home order was relaxed beginning in April, and businesses began to slowly reopen. As a result, the city experienced a surge in the number of COVID-19 cases, especially in early June 2020. ${ }^{[16]}$

The increasing psychiatric volumes led to a strain on resources. Most notably, the length of stay (LOS) for ED patients awaiting transfer to an inpatient psychiatric facility increased, surpassing pre-pandemic averages as the stayhome order was lifted in April 2020 (see Figure 2A). This redemonstrates the increase in utilization of emergency psychiatric services, and the acuity of those presenting who require inpatient psychiatric treatment.
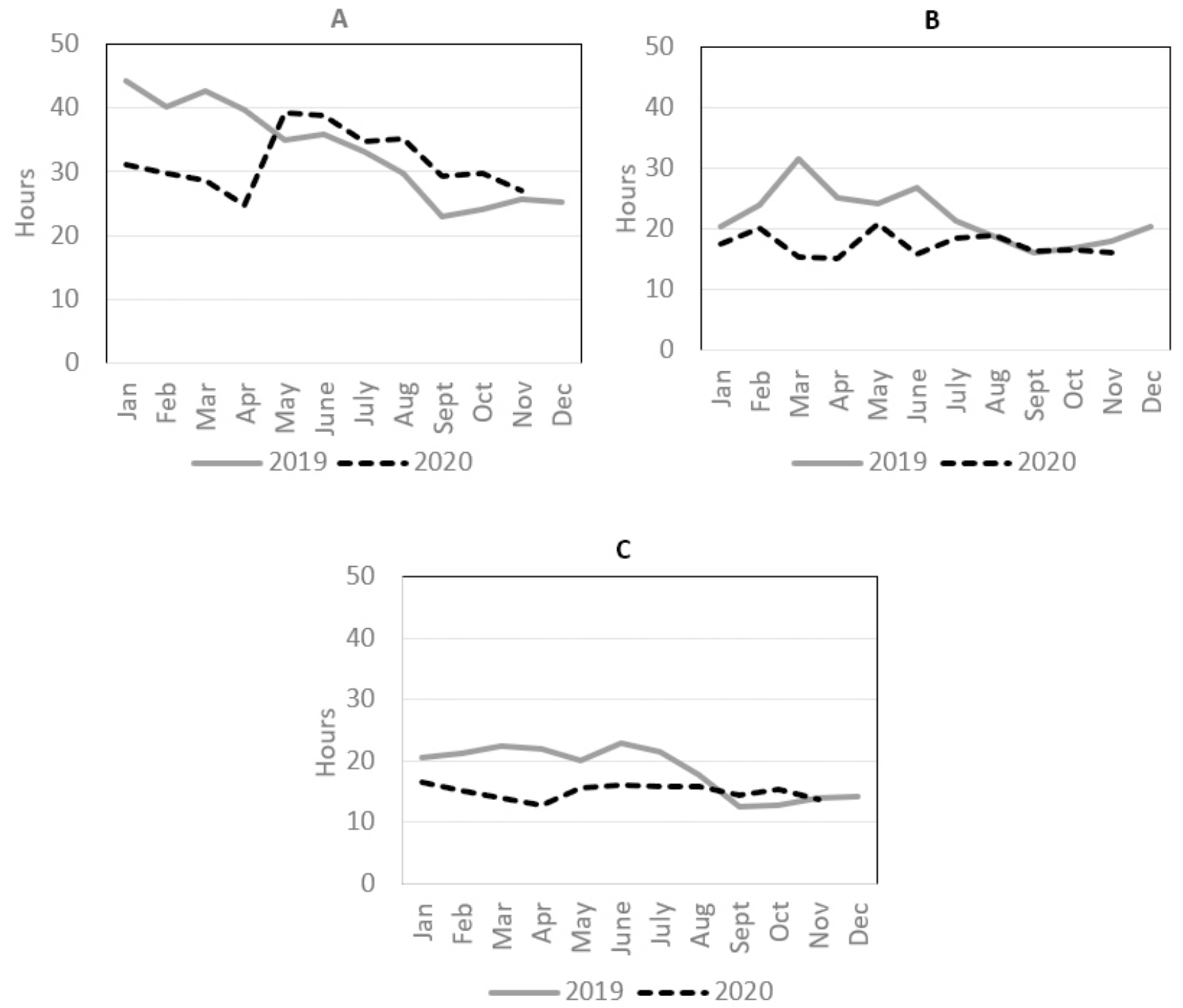

Figure 2. Length of stay for ED patients awaiting transfer to an inpatient psychiatric facility (A), length of stay for ED patients with mental-health related complaints awaiting medical beds (B), and length of stay for patients with mental-health related complaints who are eventually discharged from the ED (C). 
Interestingly, the average LOS for patients with mentalhealth related complaints who were admitted to a medicine bed (see Figure 2B), due to an active underlying medical problem that cannot be managed within an inpatient psychiatric unit, stayed consistently below or at the average from the previous year. Early on in the pandemic, the LOS for this group was lower than the trend from 2019, likely due to the overall decline in hospital volume. Patients were able to be dispositioned from the ED quickly due to ample medicine inpatient bed availability. Beginning in April 2020, as ED volume increased due to the stay-home order being lifted, the LOS did not worsen as expected. Rather, it stayed approximately the same. This may be due to the continued lower than average ED volumes when compared to pre-pandemic levels.

Those discharged from the ED (see Figure 2C) stayed consistently below the average from the previous year. As mentioned prior, patients have encountered challenges in obtaining outpatient psychiatric appointments or resources, leading to an influx of mental-health related visits in the ED, including lower acuity visits that usually warrant outpatient management. As a result, priority was placed on discharging lower acuity patients as quickly as possible from the ED to both minimize exposure risk to COVID-19 and to improve operational efficiency.

In this paper, the three levels of intervention implemented in managing the increased number of mental-health related visits during the COVID-19 pandemic will be discussed: 1) emergency department, 2) hospital-wide, 3) city-wide.

\subsection{Emergency department modifications}

The need to screen and treat potential COVID-19 infection in parallel with psychiatric symptoms has imposed new challenges to the management of these patients within the ED. Individuals with a mental disorder are at increased risk for COVID-19 infection, which is further exacerbated among certain groups such as African Americans and women. ${ }^{[17]}$ This evidence highlights the need to identify and address modifiable vulnerability factors for COVID-19 infection and to prevent delays in health care delivery. Concurrently, provisions must be made to minimize exposure and ensure safety of clinical staff and practitioners.

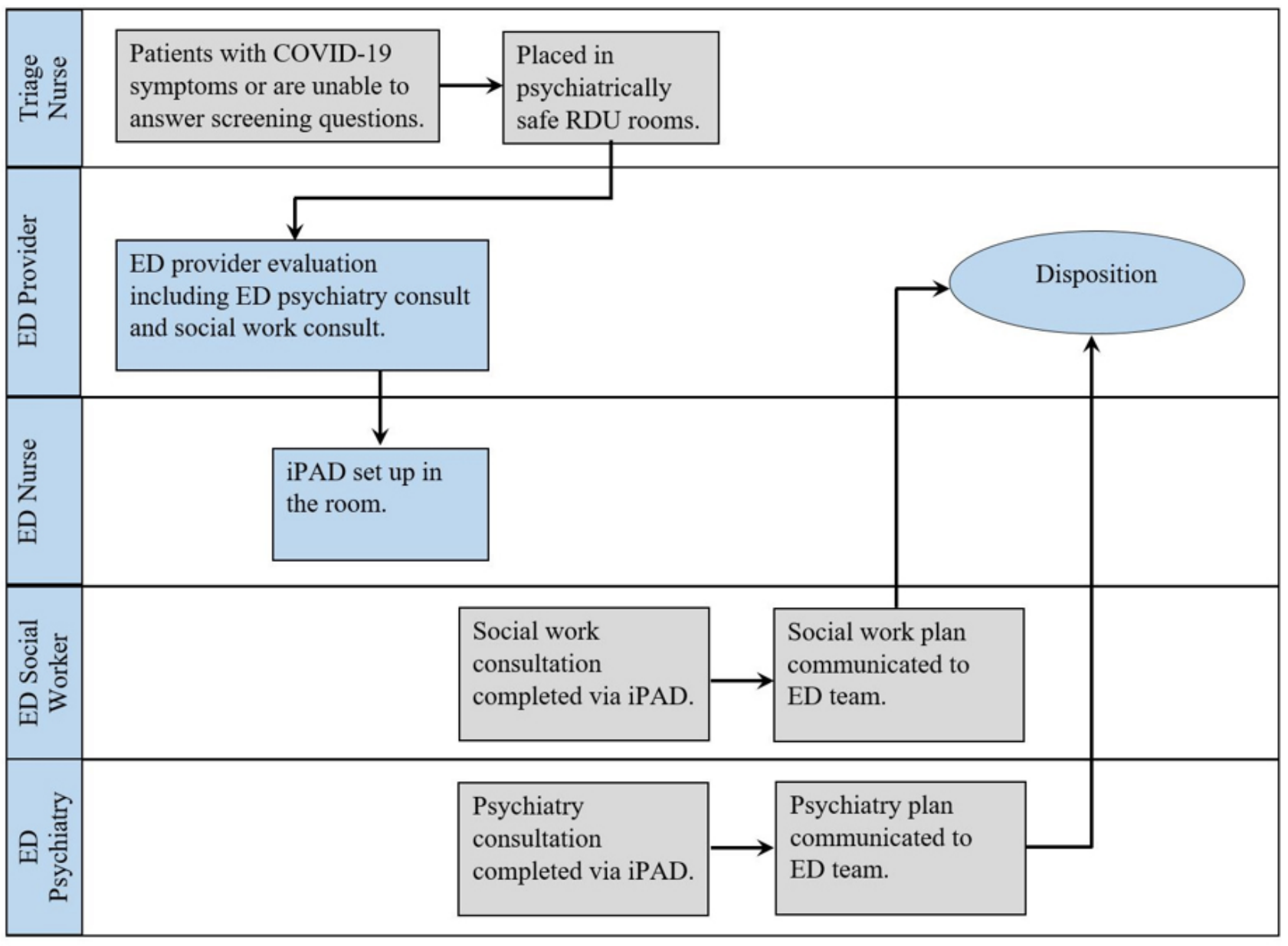

Figure 3. Triage process for patients presenting to the ED with mental-health related complaints 
Changes were implemented from the first point of contact for patients who arrived to the ED triage. A triage algorithm (see Figure 3) was created by which mental health patients who had signs and symptoms concerning for COVID-19 or who were unable to answer screening questions were triaged to the RDU. The RDU has 24 rooms, with the capability to increase capacity to 32 beds. Two climate-controlled tents were also added for an additional 12 beds, though patients with mental health complaints were not placed in the tents since they did not have private rooms.

Given that the RDU is in a different location than the typical close observation pod from the pre-pandemic care areas, the rooms were reconfigured to ensure the safety of patients with mental-health complaints. The heavy metal doors without any form of viewing window were removed from traditionally private rooms and replaced with zippered clear plastic barriers, allowing patient sitters to see inside the rooms at all times while minimizing exposure. This also eliminated a potential way for patients to lock themselves in a room or harm themselves or others.

Apart from process flow challenges, the COVID-19 pandemic has introduced unique challenges in the care of patients with significant psychiatric illnesses or psychosis. These patients, who are potential PUIs, will at times refuse to follow infection prevention protocols such as mask wearing and hand washing due to their acute mental illness. ${ }^{[18]}$ Such situations increase the risk of infection to other patients and staff. To limit unnecessary face-to-face interactions, tablets and in-room phones were utilized as much as possible for the evaluation of patients. The clinical care team was also trained to utilize appropriate personal protective equipment (PPE) donning and doffing techniques through lectures, asynchronous learning modules, and multidisciplinary simulations. Reminders of proper PPE procedures were also placed in care areas for easy reference on shift.

\subsection{Hospital wide modifications}

Hospital wide modifications unique to the COVID-19 pandemic included processes for screening, testing, and placement of patients. As discussed above, patients with mentalhealth complaints were immediately screened at triage prior to being placed in the RDU or regular ED. Once an emergency psychiatric patient was determined to need admission, either for primarily psychiatric or medical reasons, COVID19 testing was required to minimize infection risk due to unique factors within inpatient psychiatric facilities. These factors included the ligature risk of masks, absence of individual accommodations, group therapies, and inability to follow infection prevention protocol such as hand washing due to severe psychiatric symptoms. Testing allowed patients to be cohorted appropriately.

In terms of placement, initially, all patients who tested positive and required inpatient psychiatric hospitalization were primarily admitted to a medicine bed with a psychiatry consult early in the pandemic. The ED operations team worked with hospital administration to increase inpatient medicalpsychiatric bed capacity. As the pandemic continued, psychiatric hospitals were able to establish dedicated COVID-19 units, allowing the transfer of patients needing psychiatric care who were found to be COVID-19 positive, but asymptomatic or with mild disease. The ability to transfer to a COVID-19 unit at a psychiatric hospital reduced the need for unnecessary inpatient medical-psychiatric hospitalizations at our hospital. If a patient refused testing, they were admitted to the inpatient medicine floor with a psychiatry consult until they could be medically cleared.

Another unique aspect of testing and placement was the approach with emergency psychiatric patients placed in the RDU. These patients either had a positive screen at triage or were acutely psychotic and agitated and unable to answer questions. They were placed in the RDU due to their unknown status and potential risk for transmission. The ability to efficiently screen, test, and place patients in the appropriate care areas allowed for effective management of the surge in emergency psychiatric visits during the pandemic.

\subsection{City wide modifications}

Mental health services were primarily provided by three distinct entities across the city: 1) state agencies, 2) mental health agencies funded by the county, and 3) the county's primary psychiatric center which is a large scale psychiatric inpatient facility. Additionally, insurance-funded care in the private sector were available, although it was limited. Due to the stressors caused by the pandemic, there was a significant increase in presentation of patients with anxiety, depression, and decompensation of other mental disorders, including psychosis and substance use. ${ }^{[7-10]}$ This consequently caused a sharp decline in available inpatient psychiatric beds.

To mitigate the overwhelming surge of emergency psychiatric resources, leadership from the three primary entities providing mental health care in the city began to meet weekly, starting in May 2020 to develop a unified approach for management of patients with severe mental illness, homelessness, and indigent or uninsured status. These meetings included discussions on outpatient resources, 24-hour access to publicly funded inpatient psychiatric beds, and prescription pickup availability on weekends for indigent patients. Steps taken to ensure safer and better outcomes of these vulnerable patients included the sharing of data between the three mental 
health service entities, including daily and weekend census as well as bed availability. To ensure a safe disposition for COVID-19 positive homeless patients who were discharged, these entities also worked together to open shelters for these individuals.

The time to transfer a patient was also shared, and collaborative efforts led to minimize ED boarding for those awaiting an inpatient psychiatric facility from six to two hours. One challenge in minimizing the time to transfer was the requirement of constables for transfer of emergency psychiatric patients on emergency detention orders. Per state law, patients on an Emergency Detention Order require the presence of a constable in order to transfer. In addressing this challenge, constables were dedicated to the ED and were immediately available for patient transfers to inpatient psychiatric facilities, ensuring safety of patient and staff.

As a result of the collaborative efforts of the city-wide mental health leaders, the throughput of emergent psychiatric patients awaiting inpatient psychiatry placement noticeably improved following the initial worsening at the onset of the pandemic (see Figure 2A). The implementation of these steps led to a decrease in ED boarding times, as patients were able to be transferred more promptly. For instance, the boarding time of patients awaiting transfer for inpatient psychiatric admission in May 2020 was 39 hours, with improvement to 27 hours by November 2020. The ease and availability of scheduled outpatient appointments and of filling prescriptions especially on weekends further decreased the volume in the ED.

Though the experiences shared will hopefully emphasize the challenges of caring for individuals with emergent psychiatric needs during the COVID-19 pandemic, it is important to note the limitations. The focus of this article was on a single urban, public hospital, and the practice environment may differ at other institutions. There are also certain challenges related to rural settings that were not addressed and should be further studied. The long-term impact of the COVID-19 pandemic on emergent psychiatric management is also an area where future studies are warranted.

\section{Conclusions}

The COVID-19 pandemic has imposed new challenges in terms of increased psychosocial needs while ensuring safety of both patients and staff. Based on the experiences discussed above, a multitier approach is necessary, in close collaboration with a dedicated ED psychiatry team. At all levels, the primary focus was to screen appropriately, decrease transmission risk, and maintain throughput in the ED.

Infection control began at the earliest entry point into the ED, which is triage. Having a robust screening system allowed us to cohort potential PUIs to minimize transmission risk. Training of staff of appropriate PPE protocol through a multimodal approach has also helped with infection control. As another layer of screening, COVID-19 testing was required for all emergency psychiatric patients being admitted, either in a primary psychiatric or medicine bed.

The ED operations team met with hospital administration to ensure boarding times of admitted patients were kept to a minimum. This allowed for decompression within the ED, leaving room for medical, surgical, and trauma patients. The collaboration of city-wide officials also created community resources that reduced the use of the ED as a safety net.

The COVID-19 pandemic is affecting the management of emergency psychiatric patients in profound and constantly evolving ways. The approaches for addressing these challenges at a public academic hospital were described above. The experiences shared are to encourage dialogue, increase knowledge about the care of these vulnerable patients, and to inform policy makers about the unique and continued needs of the mental health system.

\section{CONFlicts OF INTEREST Disclosure}

The authors declare they have no conflicts of interest.

\section{REFERENCES}

[1] Hartnett KP, Kite-Powell A, DeVies J, et al. National Syndromic Surveillance Program Community of Practice. Impact of the COVID19 pandemic on emergency department visits-United States, January 1, 2019-May 30, 2020. MMWR Morb Mortal Wkly Rep. 2020; 69(23): 699-704. PMid: 32525856. https://doi.org/10.15585 /mmwr.mm6923e1

[2] Holland KM, Jones C, Vivolo-Kantor AM, et al. Trends in US Emergency Department Visits for Mental Health, Overdose, and Violence Outcomes Before and During the COVID-19 Pandemic. JAMA
Psychiatry. Published online February 2, 2021. PMid: 33533876. https://doi.org/10.1001/jamapsychiatry.2020.4402

[3] Gunnell D, Appleby L, Arensman E, et al. Suicide risk and prevention during the COVID-19 pandemic. The Lancet Psychiatry. 2020; 7(6): 468-471. https://doi.org/10.1016/S2215-0366(20)3 0171-1

[4] Czeisler MÉ, Lane RI, Petrosky E, et al. Mental health, substance use, and suicidal ideation during the COVID-19 pandemic-United States, June 24-30, 2020. MMWR Morb Mortal Wkly Rep. 2020; 69(32): 1049-1057. PMid: 32790653. https ://doi.org/10.155 
85/mmwr.mm6932a1

[5] Kolar D. Psychiatric emergency services and non-acute psychiatric services utilization during COVID-19 pandemic [published online ahead of print, 2020 Aug 8]. Eur Arch Psychiatry Clin Neurosci. 2020; 1-2. PMid: 32770363. https://doi.org/10.1007/s004 06-020-01182-3

[6] Hulsey J, Mellis A, Kelly B. COVID-19 pandemic impact on patients, families, and individuals in recovery from substance use disorders. Accessed July 20, 2020. Available from: https://www.addictionpolicy.org/post/covid-19-pan demic-impact-on-patients-families-individuals-in-r ecovery-fromsubstance-use-disorder

[7] Galea S, Merchant RM, Lurie N. The mental health consequences of COVID-19 and physical distancing: the need for prevention and early intervention. JAMA Intern Med. 2020; 180(6) 817-818. https://doi.org/10.1001/jamainternmed. 2020.1 562PMid: 32275292

[8] McGinty EE, Presskreischer R, Han H, et al. Psychological distress and loneliness reported by US adults in 2018 and April 2020. JAMA. 2020; 324(1): 93-94. PMid: 32492088. https://doi.org/10.1 $001 /$ jama. 2020.9740

[9] Kampfen F, Kohler IV, Ciancio A, et al. Predictors of mental health during the COVID-19 pandemic in the US: Role of economic concerns, health worries and social distancing. PLoS One. 2020: 15(11): e0241895. PMid: 33175894. https://doi.org/10.1371/jour nal.pone. 0241895

[10] Pfefferbaum B, North CS. Mental Health and the Covid-19 Pandemic. N Engl J Med. 2020 Aug 6; 383(6): 510-512. PMid: 32283003. https://doi.org/10.1056/NEJMp2008017

[11] Roll JM, Kennedy J, Tran M, et al. Disparities in unmet need for mental health services in the United States, 1997-2010. Psychiatr
Serv. 2013 Jan; 64(1): 80-2. PMid: 23280460. https ://doi.org/ 10.1176/appi.ps. 201200071

[12] Mechanic D. More people than ever before are receiving behavioral health care in the United States, but gaps and challenges remain. Health Aff (Millwood). 2014 Aug; 33(8): 1416-24. PMid: 25092844 https://doi.org/10.1377/hlthaff . 2014.0504

[13] Walker ER, Cummings JR, Hockenberry JM, et al. Insurance status, use of mental health services, and unmet need for mental health care in the United States. Psychiatr Serv. 2015; 66(6): 578-584. PMid: 25726980. https://doi.org/10.1176/appi.ps. 201400248

[14] Weil TP. Insufficient Dollars and Qualified Personnel to Meet United States Mental Health Needs. J Nervous and Mentl Disease. 2015; 203(4): 233-240. PMid: 25816044. https://doi.org/10.1097/ NMD . 0000000000000271

[15] McGuinness D. Read Harris County's stay-home order here. Houston Chronicle. Published online on March 24, 2020. Available from: https://www.houstonchronicle.com/news/houston-tex as/houston/article/Read-Harris-County-s-stay-hom e-order-here-15154443.php

[16] TMC. Weekly Average of Daily New COVID-19 positive Cases. Texas Medical Center. Accessed on March 9, 2021. Available from: https://www.tmc.edu/coronavirus-updates/weekl y-average-of-daily-new-covid-19-positive-cases/

[17] Wang ML, Behrman P, Dulin A, et al. Addressing inequities in COVID-19 morbidity and mortality: research and policy recommendations. Transl Behav Med. 2020; 10(3): 516-519. PMid: 32542349 https://doi.org/10.1093/tbm/ibaa055

[18] Russ MJ, Sisti D, Wilner P. When patients refuse COVID-19 testing, quarantine, and social distancing in inpatient psychiatry: clinical and ethical challenges. J Med Ethics. 2020; 46(9): 579-580. PMid: 32651254. https://doi.org/10.1136/medethics-2020-106 613 